To estimate rainfall in places that lack ground-based rain gauges, researchers rely on satellite data of atmospheric moisture, but this is notoriously inaccurate. Luca Brocca at the National Research Council in Perugia, Italy, and his colleagues developed an algorithm that calculates rainfall amounts on the basis of satellite data on soil moisture. They compared their estimates with rain-gauge data and found that their method accurately estimates rainfall in several regions around the world.

Moreover, their algorithm is better than a state-ofthe-art method at detecting light rainfall events and precipitation at high latitudes. J. Geophys. Res. Atmos. http://doi.org/sp7 (2014)

\section{MATERIALS}

\section{Graphene analogue carries current}

A self-assembling polymer that forms thin films and conducts electricity could beat graphene as a candidate material for flexible electronics.

Graphene, made of an atom-thick sheet of carbon, is flexible but cannot be used as a semiconductor in transistors because it lacks a 'band gap'. Mircea Dincă at the Massachusetts Institute of Technology in Cambridge and his colleagues mixed nickel with an organic compound called HITP and

ammonia in water to produce a graphene-like structure with the important band gap.

The ingredients selfassemble into a flat,

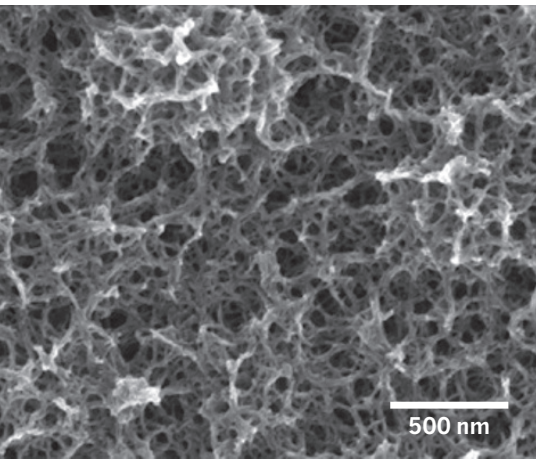

honeycomb-like structure (pictured) that has excellent electrical conductivity, unlike most other self-assembled organic-inorganic systems. The team studied the material only in bulk form, but say that the results could be even better if the polymer was in two-dimensional sheets, perhaps leading to more efficient solar cells and supercapacitors.

J. Am. Chem. Soc. http://doi.org/ spj (2014)

\section{GENOMICS \\ When brown and polar bears split}

Polar bears evolved adaptations specific to the Arctic in fewer than 20,500 generations, and diverged from brown bears much more recently than is sometimes claimed.

Rasmus Nielsen at the University of California, Berkeley, and his colleagues sequenced the genomes of 79 polar bears (Ursus maritimus) and 10 brown bears (Ursus arctos) and found that the two species diverged between 343,000 and 479,000 years ago.

Many of the genes under the greatest selection pressure in the polar bear are associated with the cardiovascular system. In particular, this bear seems to have evolved modifications in its vascular system that allow the animal to tolerate an extremely fatty diet made up mostly of blubbery seal meat.

Cell http://doi.org/sp3 (2014) For a longer story on this research, see go.nature.com/zovyry

\section{ASTROPHYSICS}

\section{Big planets could alter star rotation}

Massive planets with close-in orbits - also known as hot Jupiters - may influence the rotation and surface activity of their host stars.

Katja Poppenhaeger and Scott Wolk at the HarvardSmithsonian Center for Astrophysics in Cambridge,

SOCIAL SELECTION

\title{
Maths reality check resonates online
}

Biologists of all stripes are sharing an essay by Harvard University mathematician-turned-biologist Jeremy Gunawardena that makes a sobering observation: the mathematical equations at the core of many biological models fail to reflect nature. He argues that the components of all quantitative models should be verifiable and, most of all, the conclusions should be falsifiable. Or, in his words: "Stick the model's neck out." Jason Moore, a geneticist at Dartmouth College in New Hampshire, tweeted: "This paper is so good I am actually printing it out" - high praise in the paperless age. BMC Biol. 12, 29 (2014)

\section{9 Based on data from altmetric.com. Altmetric is supported by Macmillan Science and Education, which owns Nature Publishing Group. \\ DNATURE.COM \\ For more on popular papers: go.nature.com/mpqjve}

Massachusetts, analysed the emissions of binarystar systems, in which only one of the two stars in the system hosted an exoplanet. Comparing the differences between the emissions of the stars in each pair allowed the authors to measure the influence of the exoplanet on its host star. Using X-ray data from the Chandra and XMMNewton space telescopes, the researchers found that the stars hosting hot Jupiters showed more magnetic activity than their planet-free companions.

Magnetic activity increases with rotation, so the authors suggest that the gravitational influence of the hot Jupiters may have counteracted the natural slowing of their host stars' spin over time.

Astron. Astrophys. 565, L1 (2014)

\section{BIOLOGY}

\section{Thyroid makes young hearts grow}

A surge of thyroid hormone just before adolescence causes mouse hearts to grow drastically, suggesting that the organ may be easier to regenerate than previously thought.

Ahsan Husain of Emory

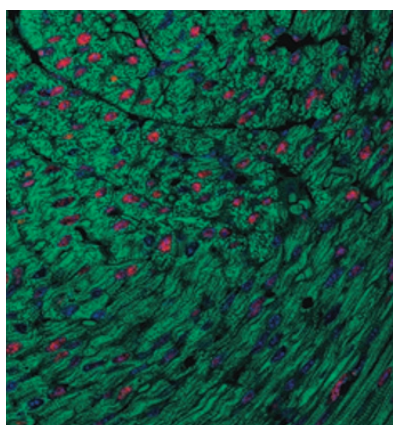

University School of Medicine in Atlanta, Georgia, and Robert Graham of the Victor Chang Cardiac Research Institute in Sydney, Australia, and their colleagues labelled heart muscle cells of baby mice with a chemical. When the mice were 15 days old, the number of cardiomyocytes (pictured, red) increased by about $40 \%$.

It had previously been thought that cardiomyocytes stopped replicating just after birth. The findings suggest that giving thyroid hormone to babies with heart defects might help to repair the organ.

Cell 157, 795-807 (2014)

\section{DNATURE.COM}

For the latest research published by Naturevisit:

www.nature.com/latestresearch 